\title{
Measurement of the Molten Pool Image during Laser Cladding Process
}

\author{
Deng Qilin \\ School of Mechanical and Power Engineering \\ Shanghai Jiao Tong University \\ Shanghai, China \\ e-mail: dengqilin@sjtu.edu.cn
}

\section{Fu Wei}

Shanghai Baosteel Industry technology Service Co., Ltd Shanghai, China e-mail: fuwei174171@baosteel.com

\begin{abstract}
Laser cladding is mainly applied in components renovation, coating, stacking forming, etc. At present, the degree of automation of laser cladding is not very high. Machine vision technology is gradually becoming a good approach to improve the automaticity. Utilizing machine vision to measure and control the molten pool to improve the degree of automation of laser cladding has become an important research interest. Clear image of molten pool was acquired with the appropriate image grabbing installation system and together with image processing methods, the area of molten pool was calculated. Analyzed variation of molten pool area as the processing parameters changed. Discussed variation of geometry of cross section of cladding bead. Explained the relationship between variation of the molten pool areas and cross section of cladding bead, according to the captured image of molten pool. When molten pool increases, it can capture more metal powder, so area of cross section of cladding bead will increase correspondingly. With appropriate processing parameters, the areas of molten pool and cross section of cladding bead are in positive correlation.
\end{abstract}

Keywords-laser cladding; molten pool; image processing; cladding quality

\section{INTRODUCTION}

Laser cladding is a non-traditional machining technology that makes high power laser beam irradiate the surface of the substrate and the cladding coat materials simultaneously so the substrate and coating material can melt and bond together in form of metallurgical bond. It is mainly applied in components renovation, coating, stacking forming, etc. At present, the degree of automation of laser cladding is not very high. In the practical production, experienced workers adjust process parameters to control cladding quality, which is mainly based on morphology of molten pool formed during laser cladding process. Machine vision technology is gradually becoming a good approach to improve the automaticity. Utilizing machine vision to measure and control the molten pool to reduce the complex manual labor has become an important research interest. Some research on the temperature field

\author{
Chen Dianbing \\ School of Mechanical and Power Engineering \\ Shanghai Jiao Tong University \\ Shanghai, China \\ e-mail: chendianbing111@163.com
}

\section{Cao Peng \\ CSIG, DCG}

Intel Asia-Pacific Research and Development Ltd Shanghai, China

e-mail: caopeng666@gmail.com

and morphology of molten pool has been carried out at home and abroad[1-8]. And there is also some further research which has established closed-loop controlled system of laser cladding based on the molten pool measurement[9-11]. Promisingly, the control system achieves good outcome.

\section{EXPERIMENTS}

\section{A. Experiment equipment}

The laser cladding measurement system employed in this paper comprises Leiou HJ-3000 traverse multi-mode continuous $\mathrm{CO} 2$ laser machine, DPSF-3 carrier-gas powder feeder, powder nozzle, 4-axis NC worktable, image grabbing system and image processing system. The numerical control system is Siemens 802C. Cladding system is shown as Fig .1, and the positions of powder nozzle and camera are shown as Fig .2. The FPS of camera is $60 \mathrm{f} / \mathrm{s}$, and the resolution is $640 * 480$. The lens is protected by a quartz glass to prevent the particles which splashes from the molten pool with high temperature from damaging it. A neutral density filter is used to reduce intensity of the incoming light. And an $850 \mathrm{~nm}$ narrowband filter is used to acquire the $850 \mathrm{~nm}$ infrared light. By adjusting the parameters of camera and optimizing the selection of density filter and filter, molten pool image is captured with a good quality (Fig .3a).

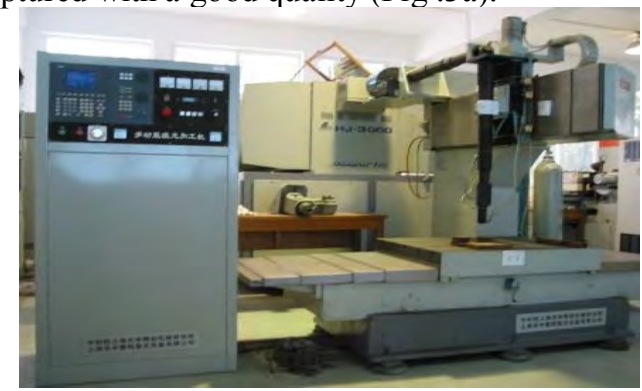

Figure 1. Laser cladding system 


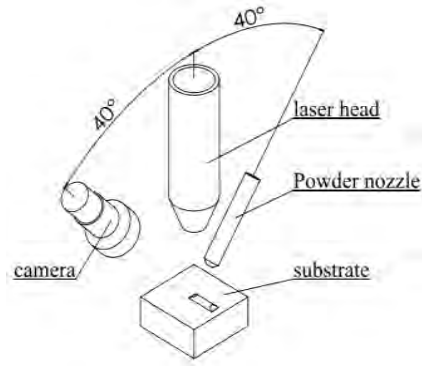

Figure 2. Camera installation

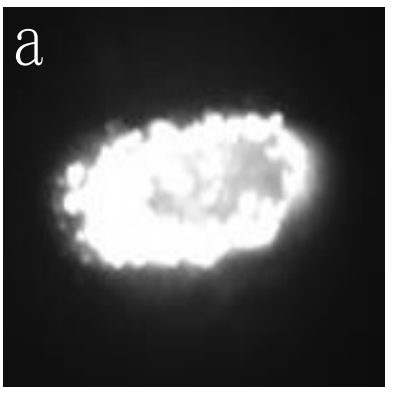

a. before processing

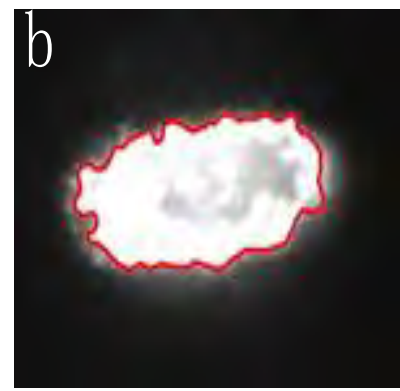

b. after processing
Figure 3. Image of molten pool

Several processing methods were applied to process the image of molten pool. Firstly, in order to remove the noise of splashing particles, low-pass space filtering was performed. Secondly, an open calculation was implanted to acquire image with smooth edge. Thirdly, gray scale gradient algorithm was taken into use to get the boundary of molten pool in the image. Finally, through calibration, the real area of the molten pool was acquired. Images before and after the processing are displayed as Fig .3a, Fig .3b. The area in the red circle is regard as molten pool.

\section{B. Experiment materials}

In the experiment, substrate is $45 \#$ steel with the size of $90 * 90 * 40 \mathrm{~mm}^{3}$. The preprocessing includes removing rust, and sand-blast on substrate surface to improve the absorptivity of laser power; the metal powder is Ni55 and the composition is displayed as TABLE 1 . Both carrier gas and protection gas is argon and gas flow is $3 \mathrm{~L} / \mathrm{min}$.

TABLE I. COMPOSITION OF METAL POWDER

\begin{tabular}{|l|c|c|c|c|c|c|}
\hline \multirow{2}{*}{ Material } & \multicolumn{6}{|c|}{ Composition } \\
\cline { 2 - 7 } & $\boldsymbol{C r}$ & $\boldsymbol{F e}$ & $\boldsymbol{C}$ & $\boldsymbol{B}$ & $\boldsymbol{S i}$ & $\boldsymbol{N i}$ \\
\hline Ni55 & $\begin{array}{l}15.0- \\
18.0\end{array}$ & 14.3 & $0.5-1.0$ & $3.5-4.5$ & $3.0-4.0$ & Bal \\
\hline
\end{tabular}

\section{Experiment process}

The experiment is performed in a way that the scanning routes are $80 \mathrm{~mm}$ with the same direction. The cladding bead is single layer. Before every cladding starts, temperature of the substrate is controlled at $200{ }^{\circ} \mathrm{C}$. By controlling the substrate temperature before cladding, the errors caused by accumulation of heat can be reduced to a lower level. The diameter of laser spot is set at $5 \mathrm{~mm}$. The processing parameters such as power, scanning velocity and power feed rate are changed to study their effect on molten pool quality.

\section{ANALYSIS OF EXPERIMENTS OUTCOME}

The head and tail of the cladding bead is unstable zone in the single bead cladding process. When analyzing the molten pool area variation as the processing parameters change, in order to reduce influence of uncertain factors, $5 \mathrm{~mm}$ from the head and $5 \mathrm{~mm}$ from the tail is taken out Only the middle part of the cladding bead which is $70 \mathrm{~mm}$ is taken into consideration. One image is taken and processed per $0.1 \mathrm{~mm}$ of the middle $70 \mathrm{~mm}$ part. According to the images, the area of molten pool is calculated and shown in Fig .4.

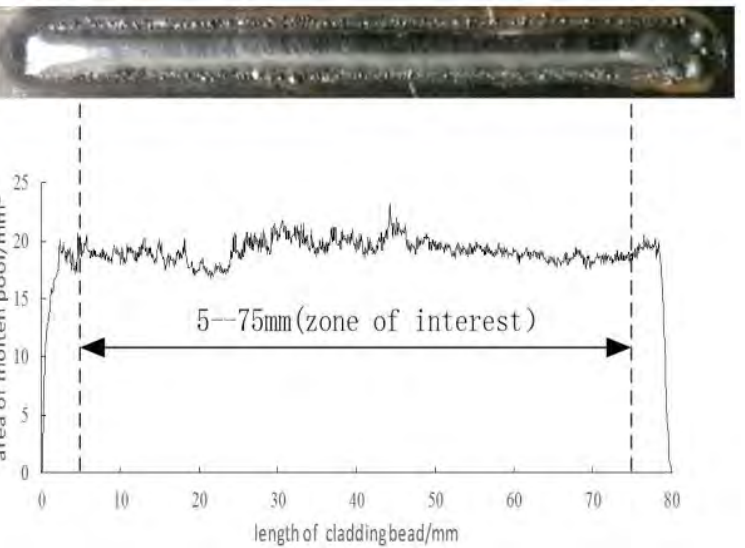

Figure 4. molten pool and morphology of cladding bead

In order to analyze the quality of the cladding, WEDM is used to acquire the cross section of cladding bead. Through sanding, polishing and etching, specimens of cladding beads under different processing parameters is made. Then, measure and compare geometries of cross section and heat affected zone under different processing parameters. Furthermore, the relationship between morphology of the molten pool and cladding bead has been analyzed in terms of power, scanning velocity, powder feeding rate.

A. Laser power

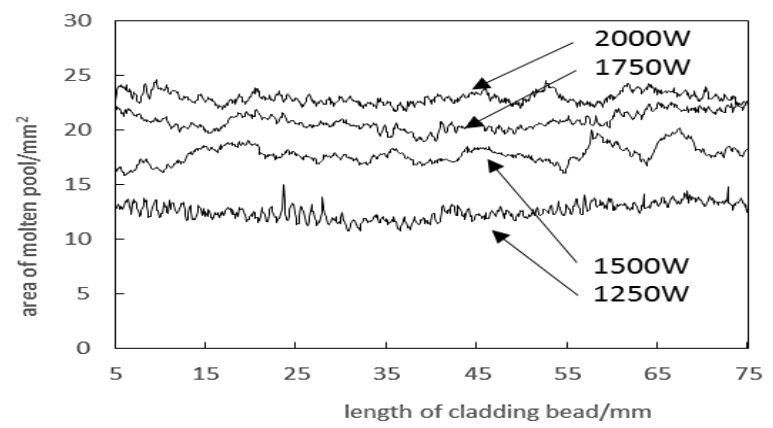

Figure 5. area of molten pool with different powers (scanning velocity $100 \mathrm{~mm} / \mathrm{min}$, powder feed rate $6 \mathrm{~g} / \mathrm{min}$ )

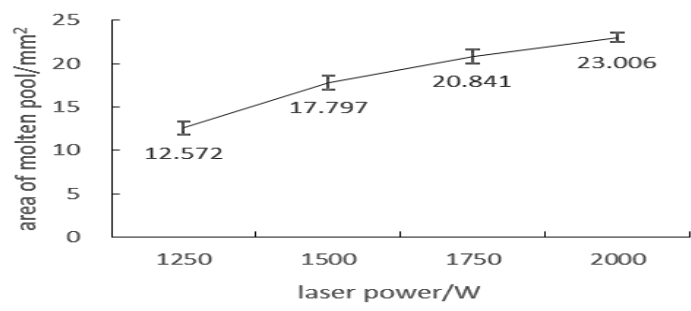

Figure 6. area of molten pool with different powers (scanning velocity $100 \mathrm{~mm} / \mathrm{min}$, powder feed rate $6 \mathrm{~g} / \mathrm{min}$ ) 


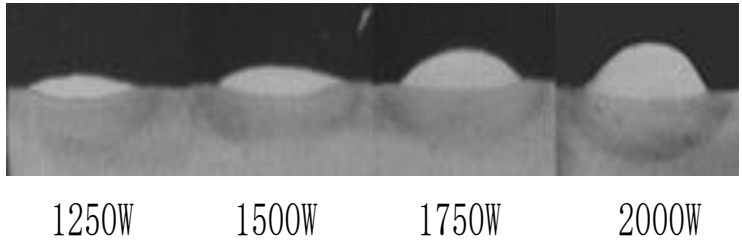

Figure 7. cross section of cladding bead with different powers (scanning velocity $100 \mathrm{~mm} / \mathrm{min}$, powder feed rate $6 \mathrm{~g} / \mathrm{min}$ )

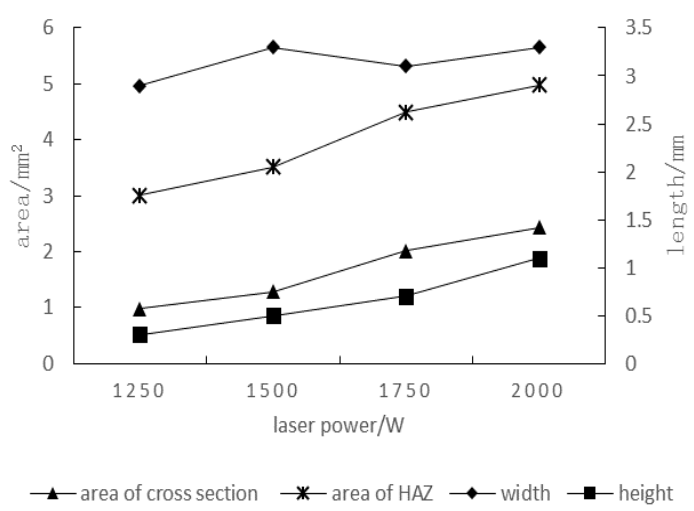

Figure 8. geometries of cross section of cladding bead with different powers (scanning velocity $100 \mathrm{~mm} / \mathrm{min}$, powder feed rate $6 \mathrm{~g} / \mathrm{min})$

From Fig .5, it can be seen that all of the 4 curves of molten pool area have the tendency to rise up. It's mainly because the pre-heated temperature $200^{\circ} \mathrm{C}$ is not very high. As the cladding proceeds, the heat will be accumulated, and the temperature will rise up and the molten pool area will increase. From the Fig .6, as the power is raised from $1250 \mathrm{~W}$ to $2000 \mathrm{~W}$, the average area of the molten pool increases from $12.572 \mathrm{~mm} 2$ to $23.006 \mathrm{~mm} 2$ with the increasing rate decreasing gradually. When power is low, area of molten pool is smaller than laser spot and is mainly decided by laser power. When laser power increases, substrate and powder absorb more heat, and more substrate gets melt, so the molten pool becomes larger. But when area of molten pool increases to a certain level closing to the laser spot area, area of molten pool is mainly decided by the laser spot area. In the experiment, the spot area doesn't increase as the laser power increase, so the increase rate of molten pool area gradually drops down.

From Fig .8, we can see, as laser power increases, the area of cross section, heat affected zone and the height of cladding bead all increases gradually. But the width of cladding beads is almost the same. It's because the width of cladding bead mainly depends on width of molten pool, hardly depending on laser power. With sufficient powder supply, the increase of area of molten pool together with laser power ascending could capture more metal powder particles. With more metal powder in the cladding bead, the area and height of cross section became larger. More laser power results in more power absorption in substrate, so the heat affected zone gets bigger.

\section{B. Scanning velocity}

From Fig .9 and 10, we can see, keeping laser power at $2 \mathrm{KW}$, powder feed rate at $6 \mathrm{~g} / \mathrm{min}$, the average area of molten pool decreases as scanning velocity rises up. The molten pool area is related to the absorbed power by substrate and powder. When scanning velocity increases, the powder absorbed per unit area of substrate decreases, which results in that substrate located in the boundary of laser spot can't melt. So the molten pool becomes smaller. When the scanning velocity increases to a certain level, it's significant to the decrease of molten pool. When scanning velocity becomes too high, the molten pool would disappear.

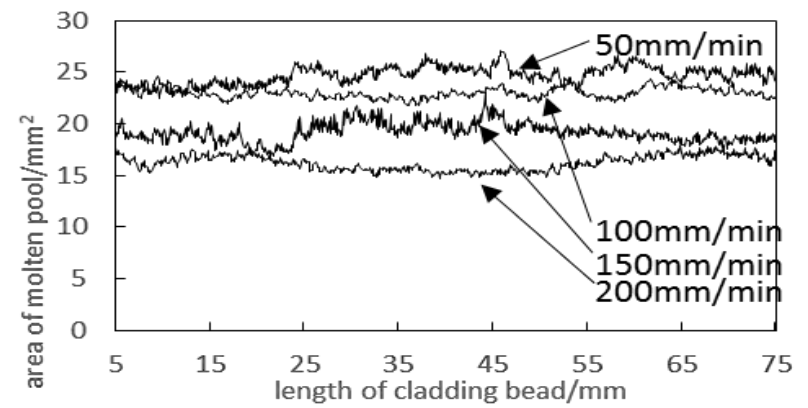

Figure 9. area of molten pool with different scanning velocities (laser power $2000 \mathrm{~W}$, powder feed rate $6 \mathrm{~g} / \mathrm{min}$ )

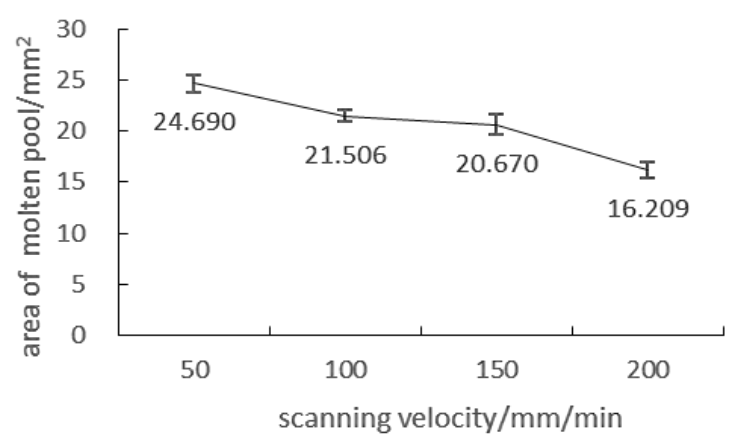

Figure 10. area of molten pool with different scanning velocities (laser power $2000 \mathrm{~W}$, powder feed rate $6 \mathrm{~g} / \mathrm{min}$ )

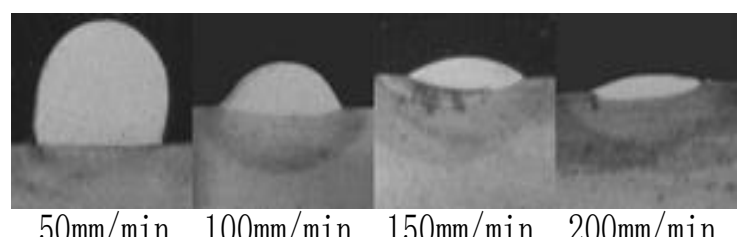

$50 \mathrm{~mm} / \mathrm{min} \quad 100 \mathrm{~mm} / \mathrm{min} \quad 150 \mathrm{~mm} / \mathrm{min} \quad 200 \mathrm{~mm} / \mathrm{min}$

Figure 11. cross section with different scanning velocities (laser power $2000 \mathrm{~W}$, powder feed rate $6 \mathrm{~g} / \mathrm{min}$ )

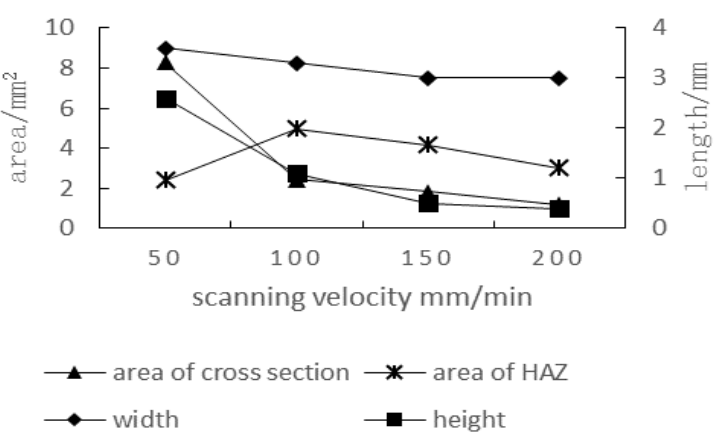

Figure 12. geometries of cross section of cladding bead with different scanning velocities (laser power $2000 \mathrm{~W}$, powder feed rate $6 \mathrm{~g} / \mathrm{min})$

As Fig .12 presents, the area and height of cross section of cladding bead decreases rapidly with the increase of scanning velocity. This's mainly because that with the 
increase of scanning velocity, absorbed power per unit length of cladding bead decreases, which makes the molten pool decrease. The decreased molten pool captures less metal powder. Besides, the scanning velocity rises up, but the powder feeding rate remains the same, which leads to less power delivery per unit length of cladding bead. As to HAZ (heat affected zone) of cross section, there is an abnormal phenomenon. In accordance with the normal condition, less power means smaller HAZ. But when scanning velocity is $50 \mathrm{~mm} / \mathrm{min}$, HAZ is very low and at $100 \mathrm{~mm} / \mathrm{min}$, it reaches the highest value. After $100 \mathrm{~mm} / \mathrm{min}$, it gradually goes down. The reason might be that, at the scanning velocity of $50 \mathrm{~mm} / \mathrm{min}$, the molten pool captures too much powder, while the powder absorbs the majority of laser power and the rest of the laser powder is not enough to melt substrate sufficiently. The bond between coat and substrate doesn't reach the metallurgical condition. Further observation of metallography confirms this analysis. The bond zone is gray instead of white. The bad bond condition couldn't convey the heat very well. The reason why HAZ becomes smaller with increase of scanning velocity is absorbed power per length unit of cladding bead decreases.

\section{Powderfeed rate}

As Fig .13 shows, keeping laser power at $2 \mathrm{KW}$, scanning velocity at $100 \mathrm{~mm} / \mathrm{min}$, curves of $6 \mathrm{~g} / \mathrm{min}$ and $12 \mathrm{~g} / \mathrm{min}$ coincide with each other at the majority part. It can also be seen that the average areas of $6 \mathrm{~g} / \mathrm{min}$ and $12 \mathrm{~g} / \mathrm{min}$ are $23.006 \mathrm{~mm}^{2}$ and $22.712 \mathrm{~mm}^{2}$ respectively with tiny difference. From Fig .14, as we can see, the area of molten pool gradually drops down when powder feed rate goes up from $3 \mathrm{~g} / \mathrm{min}$ to $9 \mathrm{~g} / \mathrm{min}$. But when the powder feed rate increases from $9 \mathrm{~g} / \mathrm{min}$ to $12 \mathrm{~g} / \mathrm{min}$, the molten pool becomes larger. When powder feed rate increases from $3 \mathrm{~g} / \mathrm{min}$ to $9 \mathrm{~g} / \mathrm{min}$, the blocking effect on the laser power reaching the substrate becomes larger. Substrate absorbs less laser power so the area of molten pool becomes smaller. But when the powder feed rate increases to a certain level, the height of cladding bead would increase because of more powder particles captured by molten pool. And the angle between the surfaces of molten pool and substrate becomes bigger. This angle would increase the molten pool area. But when this phenomenon happens, the bond condition between cladding bead and substrate becomes bad.

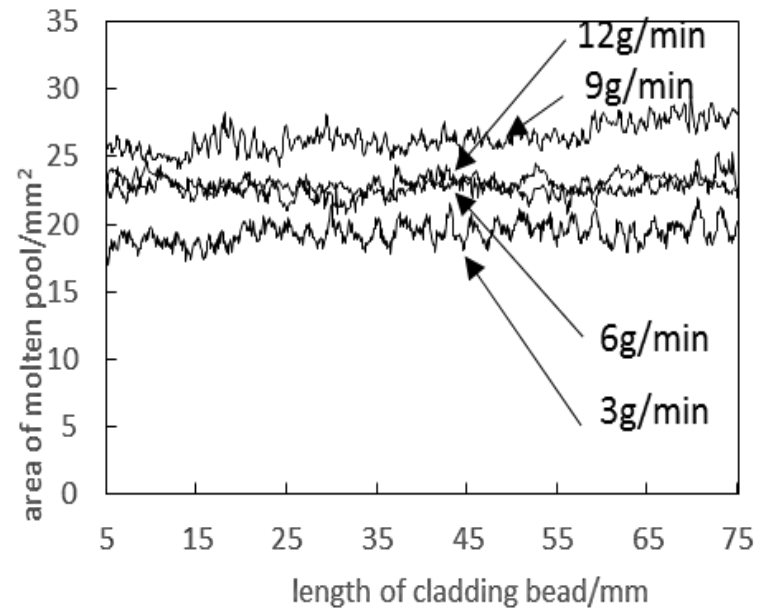

Figure 13. area of molten pool with different powder feed rates (laser power $2000 \mathrm{~W}$, scanning velocity $100 \mathrm{~mm} / \mathrm{min}$ )

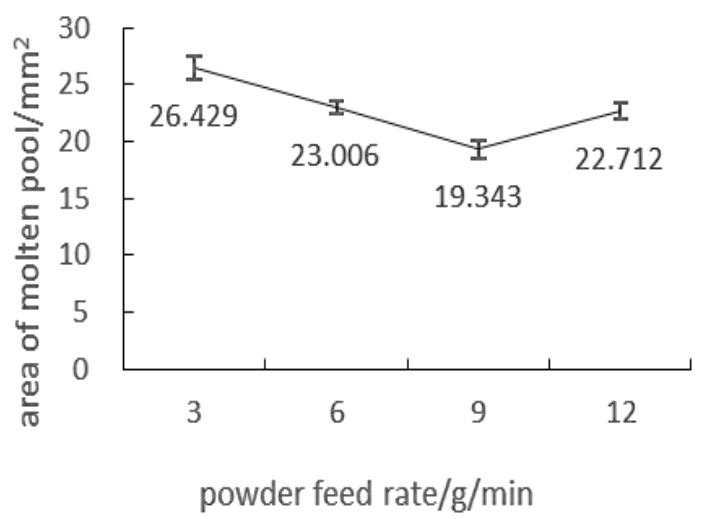

Figure 14. area of molten pool with different powder feed rates (laser power $2000 \mathrm{~W}$, scanning velocity $100 \mathrm{~mm} / \mathrm{min}$ )

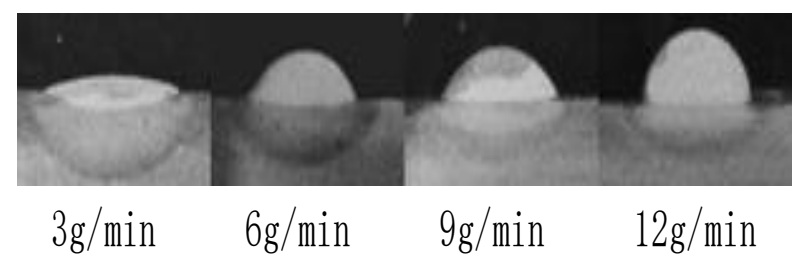

Figure 15. cross section of cladding bead with different powder feed rates (laser power $2000 \mathrm{~W}$, scanning velocity $100 \mathrm{~mm} / \mathrm{min}$ )

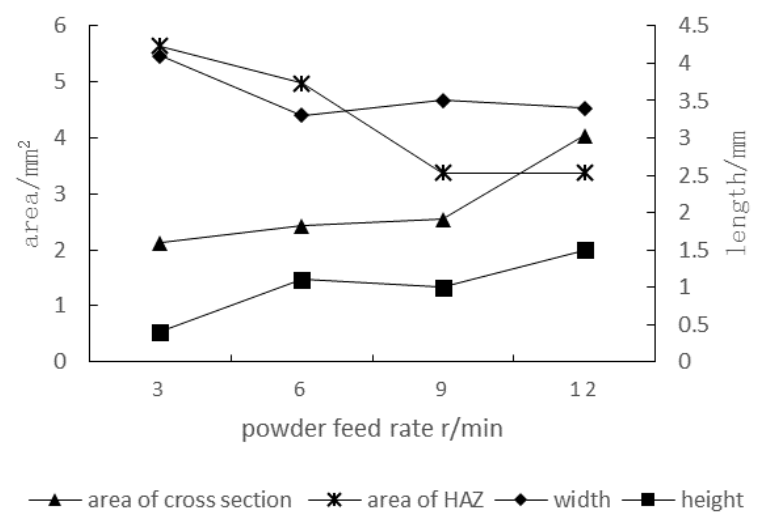

Figure 16. geometries of cross section of cladding bead with different powder feed rates (laser power 2000W, scanning velocity $100 \mathrm{~mm} / \mathrm{min}$ )

As we can see from Fig .16, the cross section area and height of cladding bead increases as powder feed rate increases; while the area of HAZ becomes smaller. The width of cladding bead drops down as powder feed rate increases from $3 \mathrm{~g} / \mathrm{min}$ to $6 \mathrm{~g} / \mathrm{min}$ and then keeps almost the same. The dropping of width of cladding bead is mainly because when powder feed rate is too small, the cladding process becomes alloy process. Most of laser power is absorbed by substrate. Hence, heat conduction is more and width of cladding bead increases.

\section{CONCLUSION}

1. Clear image of molten pool was acquired with the appropriate image grabbing installation system and together with image processing methods such as filtering, morphological processing, calibrating, the area of molten pool was calculated. 
2. Analyzed variation of molten pool area as the processing parameters (including laser power, scanning velocity, powder feed rate) changed. Generally, the area increases as laser power increases; the area decreases as scanning velocity increases; the area decreases as powder feed rate increase.

3. Discussed variation of geometry of cross section of cladding bead. Generally, the area of cross section of cladding bead increases as the laser power increases; the area of cladding bead decreases as scanning velocity increases; the area of cladding bead increases as powder feed rate increases.

4. Explained the relationship between variation of the molten pool areas and cross section of cladding bead, according to the captured image of molten pool. When molten pool increases, it can capture more metal powder, so area of cross section of cladding bead will increase correspondingly. With appropriate processing parameters, the areas of molten pool and cross section of cladding bead are in positive correlation.

\section{ACKNOWLEDGMENTS}

This research was financially supported by the National Natural Science Foundation of China (No. 51275303).

\section{REFERENCES}

[1] Doubenskaia M, Bertrand P, Smurov I. Optical monitoring of Nd: YAG laser cladding[J]. Thin Solid Films, 2004, 453477-485.
[2] Bi G, Gasser A, Wissenbach K, etc. Identification and qualification of temperature signal for monitoring and control in laser cladding $[\mathrm{J}]$. Optics and lasers in engineering, 2006, 44(12): 13481359 .

[3] Iravani-Tabrizipour M, Toyserkani E. An image-based feature tracking algorithm for real-time measurement of clad height[J]. Machine Vision and Applications, 2007, 18(6): 343-354.

[4] Jianbo Lei. Measurement of Laser Remanufacturing Molten Pool Temperature Field Based on CCD[D]. Tianjin Polytechnic University, 2007.

[5] JIANG Shu-juan,LIU Wei-jun. Study on Real-time Measurement for Laser Molten Pool Temperature Field by Images Colorimetric Method[J]. Information and control, 2008, 37(6): 747-750,756.

[6] Pavlov M, Novichenko D, Doubenskaia M. Optical Diagnostics of Deposition of Metal Matrix Composites by Laser Cladding[J]. Physics Procedia, 2011, 12674-682.

[7] Liushan Yang. Development and Application of Laser Cladding On-line Measurement System based on CCD[D]. Hunan University, 2011.

[8] Smurov I, Doubenskaia M, Zaitsev A. Comprehensive analysis of laser cladding by means of optical diagnostics and numerical simulation[J]. Surface and Coatings Technology, 2013, 220112 121.

[9] Fox M D, Hand D P, Su D, etc. Optical sensor to monitor and control temperature and build height of the laser direct-casting process[J]. Applied optics, 1998, 37(36): 8429-8433.

[10] Hu D, Kovacevic R. Sensing, modeling and control for laser-based additive manufacturing $[\mathrm{J}]$. International Journal of Machine Tools and Manufacture, 2003, 43(1): 51-60.

[11] Salehi D, Brandt M. Melt pool temperature control using LabVIEW in Nd: YAG laser blown powder cladding process[J]. The International Journal of Advanced Manufacturing Technology, 2006 , $29(3-4)$ : 273-278. 